\title{
The AMBRE project: A new synthetic grid of high-resolution FGKM stellar spectra
}

\author{
P. de Laverny ${ }^{1}$, A. Recio-Blanco ${ }^{1}$, C. C. Worley $^{1}$, and B. Plez ${ }^{2}$ \\ ${ }^{1}$ Laboratoire Lagrange, UMR 7293, Université de Nice Sophia Antipolis, CNRS, Observatoire de la Côte d'Azur, BP 4229, \\ 06304 Nice Cedex 4, France \\ e-mail: laverny@oca.eu \\ 2 Laboratoire Univers et Particules de Montpellier, Université Montpellier II, CNRS, 34095 Montpellier Cedex 05, France
}

Received 2 April 2012 / Accepted 9 May 2012

\section{ABSTRACT}

\begin{abstract}
Context. Large grids of synthetic spectra covering a wide range of stellar parameters are essential tools for different stellar and (extra-) Galactic physical applications. They can be used for the automatic parametrisation of stellar spectra such as that performed by the AMBRE project whose main goal is to determine the stellar atmospheric parameters of a few hundred thousands of archived spectra of four ESO spectrographs.

Aims. To fulfil the needs of AMBRE and similar future projects, we computed a grid of synthetic spectra over the whole optical domain for cool to very cool stars of any luminosity (from dwarfs to supergiants) with metallicities varying from $10^{-5}$ to 10 times the solar metallicity, and considering large variations in the chemical composition of the $\alpha$-elements.

Methods. In these spectrum computations, we used new generation MARCS model atmospheres and the Turbospectrum code for radiative transfer. We also took into account atomic and molecular linelists that were as complete as possible and adopted, in the spectral synthesis, the same physical assumptions and input data as in the MARCS models. This allowed us to present a grid in which there is a high consistency between the atmosphere models and the synthetic spectra.

Results. We present a new grid of 16783 high resolution spectra over the wavelength range $3000 \AA$ to $12000 \AA$ computed at a spectral resolution higher than 150000 . Normalised and absolute flux versions are available over a wide range of stellar atmospheric parameters for stars of FGKM spectral types. The parameters covered are $2500 \mathrm{~K} \leq T_{\text {eff }} \leq 8000 \mathrm{~K},-0.5 \leq \log (g) \leq 5.5$ dex, and $-5.0 \leq[\mathrm{M} / \mathrm{H}] \leq+1.0$ dex. Moreover, for each combination of these stellar parameters, five different values of the enrichment in $\alpha$-elements were considered $(0.0, \pm 0.2 \mathrm{dex}$ and $\pm 0.4 \mathrm{dex}$ around the standard values). This library is thus relevant to any stellar type and luminosity class, present in old and intermediate-age stellar populations with chemical composition varying from extremely metal-poor to metal-rich. This grid is made publicly available through the POLLUX database (about 50\% of the spectra have been included in this database) and in FITS format upon request to the authors.
\end{abstract}

Key words. stars: atmospheres - stars: fundamental parameters - stars: abundances - astronomical databases: miscellaneous surveys

\section{Introduction}

AMBRE is a collaborative project between the Observatoire de la Côte d'Azur and the European Southern Observatory (ESO). Its main goal is the automated stellar parametrisation of FEROS, HARPS, UVES, and GIRAFFE archived stellar spectra. This parametrisation of more than 300000 spectra will be made publicly available through the ESO archive. Furthermore, AMBRE offers the opportunity to test on a substantial number of real spectra the performances of parametrisation algorithms such as MATISSE (Recio-Blanco et al. 2006) or DEGAS (Kordopatis et al. 2011), which have been specifically developed for the analysis of Radial Velocity Spectrometer (RVS) data collected by the ESA/Gaia mission. We refer the reader to Worley et al. (2012) for a detailed description of AMBRE and the complete analysis of the FEROS spectra.

Within the AMBRE project, the adopted algorithms for the automatic parametrisation of stellar spectra rely on a library of reference spectra to derive the atmospheric parameters and the chemical abundances. Preparing such a spectra grid, covering a wide range of parameters is mandatory in order to allow a rapid analysis with specially developed algorithms of large quantities of observed spectra from any stellar type, such as those provided by current (RAVE, SEGUE, Gaia/ESO Survey...) and future (Gaia/RVS) spectroscopic surveys. Therefore, while planning AMBRE, libraries of observed (empirical) and/or computed (synthetic) stellar spectra were investigated. We ultimately concluded that there is a lack of an extensive library of stellar spectra with the following properties: (i) coverage of a wide range of atmospheric parameters; (ii) coverage of a very large spectral domain in the optical; (iii) high spectral resolution; (iv) well-established atmospheric parameters; and (v) inclusion of chemical abundance variations.

Although important progress has been made over the past few years to build high-quality empirical libraries of stellar spectra (see, in particular, the last release of the CFLIB library by Wu et al. 2011), observed spectra libraries still often have a number of severe shortcomings. For instance, most of them rely on rather low resolution spectra (CFLIB or MILES, Vazdekis et al. 2010) or rather restricted wavelength coverage (as the ELODIE library, Prugniel \& Soubiran 2001) and/or suffer from flux calibration uncertainties. Furthermore, the atmospheric parameters attached to these compiled stellar spectra may be uncertain owing to the lack of determination of some parameters and the different adopted bibliographic sources and/or method of determination (see Soubiran et al. 2010). However, there have been many 
efforts similar to that recently conducted by Wu et al. (2011) to obtain a homogeneous set of stellar atmospheric parameters, even though this new CFLIB library still suffers from a rather incomplete coverage of the parameter space (particularly for cool and/or metal-poor stars). Furthermore, no information on the chemical abundance of $\alpha$ elements is provided (to our knowledge, all available empirical libraries provide only the main three, in the best cases, atmospheric parameters, i.e. the effective temperature, the surface gravity and the mean metallicity). All of these reasons have prevented the use of these empirical libraries within AMBRE because, as mentioned previously, the main goal of AMBRE is to derive from high-resolution spectra covering very different regions of the whole optical domain, the stellar atmospheric parameters together with the $[\alpha / \mathrm{Fe}]$ enrichment of the target stars for the purposes of galactic archaeology.

The synthetic spectra libraries available to us were also not completely satisfactory. Munari et al. (2005) present an extensive grid of cool to very hot stars based on Kurucz/ATLAS9 model atmospheres and the SYNTHE spectral synthesis code (see also their Table 1 for a comparison of the principal characteristics of most publicly available libraries before 2005). Although the number of available spectra is impressive (because the authors considered different values of the microturbulent and rotational velocities, spectral resolution, and sampling law), these spectra actually correspond to merely a few thousand combinations of $T_{\text {eff }}, \log (g)$, and $[\mathrm{M} / \mathrm{H}]$ values. Furthermore, the highest spectral resolution $(R \sim 20000)$ is too low for the analysis of the ESO spectra and, as for the empirical libraries, no variations of the $[\alpha / \mathrm{Fe}]$ enrichment have been considered (two values of $[\alpha / \mathrm{Fe}]$ were computed at only three distinct metallicities). Finally, the metallicity range is also too small for general parametrisation $(-2.5 \leq[\mathrm{M} / \mathrm{H}] \leq 0.5)$. Another synthetic high-resolution spectra grid was presented by Coelho et al. (2005), which is again based on Kurucz/ATLAS9 models. This grid covers the whole optical domain but, once again, the adopted variations in $[\alpha / \mathrm{Fe}]$ are still unsatisfactory (only $[\alpha / \mathrm{Fe}]=0$ and 0.4 dex are considered in every model) for the automatic parametrisation purposes for which this chemical index is searched. Finally, Kirby (2011) published a grid very similar to our needs in terms of atmospheric parameter ranges but, unfortunately, it is dedicated to the red part of the optical domain $(\lambda>6300 \AA)$. These spectra are computed from a new grid of Kurucz/ATLAS9 atmosphere models and the MOOG code. There is however a lack of molecular lines considered in the spectral synthesis. Only the $\mathrm{CN}, \mathrm{C}_{2}$, and $\mathrm{MgH}$ molecules were considered (partly because the number of TiO lines to be considered led to prohibitive computational times). Therefore, the provided spectra for cool and/or metal-rich stars might not be well modelled in this grid.

For all these reasons, we decided to compute a new synthetic grid of stellar spectra in order to fulfil the objectives of AMBRE. The goal of this paper is to present and make such a grid publicly available. The computed spectra cover the whole optical spectral range (from $3000 \AA$ to $12000 \AA$ ) at high resolution $(R>150000)$ in order to be used for the analysis of most spectrograph data. They correspond to FGKM stars of any luminosity (except cool white dwarfs) with a very wide range of metallicities and $\alpha$-element abundance variations at any metallicity. Furthermore, it is the first grid based exclusively on MARCS model atmospheres and associated tools to compute the synthetic spectra (with therefore the same physical assumptions and ingredients, such as the extensive molecular linelists). To date, it is also the most extensive grid by about one order of magnitude in terms of the number of available spectra of cool stars (mostly because we span cooler temperatures, lower metallicities, and several variations in $[\alpha / \mathrm{Fe}])$. This grid can easily be used as a reference for the automatic determination of atmospheric parameters (as carried out in the AMBRE project) or for building composite synthetic stellar populations for extragalactic studies. In particular, the simulation of the integrated spectra of dwarf galaxies similar to those found in the Milky Way vicinity that contain stars with large or small $[\alpha / \mathrm{Fe}]$ values (Frebel et al. 2010; Letarte et al. 2010) requires such a library with a broad range of $[\alpha / \mathrm{Fe}]$ chemical enrichments. This is also mandatory for future and present Galactic spectroscopic surveys searching for accreted halo stars.

This paper is organised as follow: Sect. 2 is devoted to the description of the methods, assumptions, and input data adopted for generating the stellar spectra. The AMBRE grid of synthetic spectra is then presented in Sect. 3. Sections 4 and 5 discuss some properties and limitations of this grid. We present in Sect. 6 how these spectra can be retrieved. The conclusions are given in Sect. 7 .

\section{Computation of the synthetic spectra}

The AMBRE project is primarily devoted to the analysis of FGKM stars, i.e. the cool stars that have been the main motivation behind the development of the MARCS model atmospheres for almost 40 years (Gustafsson et al. 1975). Therefore, the presented grid is based on the last generation of MARCS model atmospheres presented in Gustafsson et al. (2008). The synthetic spectra were generated in a consistent way (as far as possible) with these models. The spectra were indeed computed with the Turbospectrum code for spectral synthesis V9.2 (Alvarez \& Plez 1998, with subsequent improvements through the years by Plez). This code uses the same routines and input data as the MARCS code. We recall that MARCS models were computed assuming one-dimensional plane-parallel or spherical geometry (depending on the gravity, see below), hydrostatic and local thermodynamic equilibria, and convection treated following the mixing-length theory.

We have seen that all the other available grids of synthetic spectra are based on Kurucz/ATLAS9 model atmospheres. Gustafsson et al. (2008) compared their new generation MARCS models with the ATLAS9 ones (see also, Plez 2011). They reported a rather good agreement in the temperature structures for solar-type or cooler dwarf stars of different metallicities. However, larger discrepancies appear at lower $T_{\text {eff }}$ and/or lower $[\mathrm{M} / \mathrm{H}]$. Furthermore, for giants, MARCS models appear slightly cooler in the upper layers (probably owing to the improved molecular data collected by the MARCS group over the years). The presented AMBRE spectra grid is therefore expected to represent an important improvement towards the modelling of cool stars of any luminosity (from dwarfs to supergiants) and metallicity.

\subsection{Atomic and molecular line opacities}

In the spectrum calculations, a number of specific line lists for hydrogen, metals, and molecules covering the 3000-12000 spectral range were considered.

Atomic line data were recovered from the Vienna Atomic Line Database (its August 2009 version; Kupka et al. 1999). Only lines from neutral or singly ionised atoms were kept (which is a reasonable assumption for the considered effective temperatures). This led to a total of about 215000 atomic 
P. de Laverny et al.: The AMBRE grid of cool star synthetic spectra

Table 1. Isotopic compositions adopted in the calculations of the molecular equilibrium (see, for references, the Turbospectrum code).

\begin{tabular}{|c|c|c|c|c|c|c|c|c|c|c|}
\hline Element & & & & & Isot & pes & & & & \\
\hline $\mathrm{C}$ & ${ }^{12} \mathrm{C}$ & $98.90 \%$ & ${ }^{13} \mathrm{C}$ & $1.10 \%$ & & & & & & \\
\hline $\mathrm{N}$ & ${ }^{14} \mathrm{~N}$ & $99.634 \%$ & ${ }^{15} \mathrm{~N}$ & $0.366 \%$ & & & & & & \\
\hline $\mathrm{O}$ & ${ }^{16} \mathrm{O}$ & $99.762 \%$ & ${ }^{17} \mathrm{O}$ & $0.038 \%$ & ${ }^{18} \mathrm{O}$ & $0.20 \%$ & & & & \\
\hline $\mathrm{Mg}$ & ${ }^{24} \mathrm{Mg}$ & $78.99 \%$ & ${ }^{25} \mathrm{Mg}$ & $10.00 \%$ & ${ }^{26} \mathrm{Mg}$ & $11.01 \%$ & & & & \\
\hline $\mathrm{Si}$ & ${ }^{28} \mathrm{Si}$ & $92.23 \%$ & ${ }^{29} \mathrm{Si}$ & $4.67 \%$ & ${ }^{30} \mathrm{Si}$ & $3.10 \%$ & & & & \\
\hline $\mathrm{Ca}$ & ${ }^{40} \mathrm{Ca}$ & $96.941 \%$ & ${ }^{42} \mathrm{Ca}$ & $0.647 \%$ & ${ }^{43} \mathrm{Ca}$ & $0.135 \%$ & ${ }^{44} \mathrm{Ca}$ & $2.086 \%$ & ${ }^{48} \mathrm{Ca}$ & $0.187 \%$ \\
\hline $\mathrm{Ti}$ & ${ }^{46} \mathrm{Ti}$ & $8.00 \%$ & ${ }^{47} \mathrm{Ti}$ & $7.30 \%$ & ${ }^{48} \mathrm{Ti}$ & $78.30 \%$ & ${ }^{49} \mathrm{Ti}$ & $5.50 \%$ & ${ }^{50} \mathrm{Ti}$ & $5.40 \%$ \\
\hline V & ${ }^{50} \mathrm{~V}$ & $0.25 \%$ & ${ }^{51} \mathrm{~V}$ & $99.75 \%$ & & & & & & \\
\hline $\mathrm{Fe}$ & ${ }^{54} \mathrm{Fe}$ & $5.80 \%$ & ${ }^{56} \mathrm{Fe}$ & $91.72 \%$ & ${ }^{57} \mathrm{Fe}$ & $2.20 \%$ & ${ }^{58} \mathrm{Fe}$ & $0.28 \%$ & & \\
\hline $\mathrm{Zr}$ & ${ }^{90} \mathrm{Zr}$ & $51.46 \%$ & ${ }^{91} \mathrm{Zr}$ & $11.22 \%$ & ${ }^{92} \mathrm{Zr}$ & $17.15 \%$ & ${ }^{94} \mathrm{Zr}$ & $17.38 \%$ & ${ }^{96} \mathrm{Zr}$ & $2.80 \%$ \\
\hline
\end{tabular}

Table 2. Atmospheric parameters of the AMBRE grid.

\begin{tabular}{lccccc}
\hline \hline Atmospheric parameter (units) & Minimum & Maximum & Step & & Number of possible values \\
\hline$T_{\text {eff }}$ (in K) & 2500 & 8000 & 200 & for $T_{\text {eff }} \leq 3900$ & 25 \\
& & & 250 & for $T_{\text {eff }} \geq 4000$ & 13 \\
$\log (g)\left(\right.$ in cm/s $\left.\mathrm{s}^{2}\right)$ & -0.5 & +5.5 & 0.5 & & 15 \\
{$[\mathrm{M} / \mathrm{H}]$ (in dex) } & -5.0 & +1.0 & 0.25 & for $[\mathrm{M} / \mathrm{H}] \geq-1.0$ & \\
& & & 0.5 & for $-3.0 \leq[\mathrm{M} / \mathrm{H}]<-1.0$ & 5 for a given $[\mathrm{M} / \mathrm{H}]$ \\
\hline$\alpha / \mathrm{Fe}]$ (in dex) & & & 1.0 & for $[\mathrm{M} / \mathrm{H}]<-3.0$ & \\
\hline
\end{tabular}

Notes. A description of the other parameters adopted in the spectra calculation is given in Sects. 2 and 3. We also recall that (i) for each set of $\left(T_{\text {eff }}, \log (g)[\mathrm{M} / \mathrm{H}]\right)$ values, five spectra with different $[\alpha / \mathrm{Fe}]$ enrichments have been computed, and that (ii) some models are missing (approach to the Eddington flux limit and/or poor convergence).

lines from 76 neutral metals and 60 singly ionised metals (among those lines, about 35100 of them are transitions of Fe I and 33600 of Fe II). Owing to the huge amount of adopted lines over the wide spectral range, neither a correction nor astrophysical calibration of the atomic data was performed (the completeness and the quality of the VALD linelists could be questionned, although this database continues to be improved thanks to huge efforts conducted by several groups). Therefore, we warn future users of this synthetic spectra grid that it is not optimised for fine analysis or abundance determinations based on fitting individual spectral lines. As in Gustafsson et al. (2008), hydrogen selfbroadening is estimated following Barklem et al. (2000a) and damping constants for many strong metallic lines are calculated using recent data (Barklem et al. 2000b, and related references) ${ }^{1}$. Other lines are broadened with the classical Unsöld theory with an assumed enhancement factor for the Van der Waals broadening set to 2.5, except for Na I, Si I, Ca I, and Fe I for which the enhancement factor was set to $2.0,1.3,1.8$, and 1.4 , respectively (see Gustafsson et al. 2008).

The considered molecular lines come from the $\mathrm{CH}, \mathrm{NH}, \mathrm{OH}$, $\mathrm{MgH}, \mathrm{SiH}, \mathrm{CaH}, \mathrm{FeH}, \mathrm{C} 2, \mathrm{CN}, \mathrm{TiO}, \mathrm{VO}$, and $\mathrm{ZrO}$ molecules with their corresponding isotopic variations. In these calculations, we disregarded CO lines since they appear beyond the infrared wavelength limit of the present grid at $\lambda>1.5$ microns. We however point out that this molecule together with several other molecular species have been considered in the calculation of the chemical equilibrium (see the complete list in Gustafsson et al. 2008). In total, 41 different species (see Table 1 for the

\footnotetext{
${ }_{1}$ One can expect some differences (particularly in the H-line profiles) in our computed fluxes when compared to previous grids since these improvements have never been considered until now by other groups computing large spectra grids.
}

adopted isotopic ratios) consisting of more than 20 million molecular lines were taken into account (half of these lines being $\mathrm{TiO}$ transitions and one fourth belong to the $\mathrm{ZrO}$ molecule). These molecular line lists have been compiled by Plez and are identical to those used by Gustafsson et al. (2008, see their Table 2 for a list of references). We point out that, for molecular lines, the damping was not taken into account for the same reasons as in the MARCS models.

\subsection{Selection of MARCS model atmospheres}

We recovered 3358 MARCS model atmospheres (version of September 2009; only converged models were kept). The two main atmospheric parameters of the selected models (see Table 2) are the effective temperature $\left(T_{\text {eff }}\right)$ and the stellar surface gravity $(\log (g))$. The effective temperatures vary between $2500 \mathrm{~K}$ and $8000 \mathrm{~K}$ (in steps of $200 \mathrm{~K}$ below $4000 \mathrm{~K}$ and $250 \mathrm{~K}$ above) and the logarithm of stellar surface gravities from -0.5 dex to $5.5 \mathrm{dex}$ (in steps of $0.5 \mathrm{dex}$ ), $g$ in units of $\mathrm{cm} / \mathrm{s}^{2}$.

Then, the selected models cover the mean metallicity $([\mathrm{M} / \mathrm{H}]$ hereafter, except for the $\alpha$-elements) range from -5.0 to +1.0 , spanning 15 different values (see Figs. 1, 2). We point out that, throughout this paper and the AMBRE project, the mean metallicity refers to solar-scaled abundances of all elements heavier than He. We note that the adopted metallicity range allows for the analysis of very different types of Galactic stellar populations, including extremely metal-poor halo stars.

Finally, a selection has been performed on the enrichment in $\alpha$-elements. As in Gustafsson et al. (2008), the chemical species $\mathrm{O}, \mathrm{Ne}, \mathrm{Mg}, \mathrm{Si}, \mathrm{S}, \mathrm{Ar}, \mathrm{Ca}$, and $\mathrm{Ti}$ are considered as $\alpha$-elements and we consider that they vary in lockstep. We recall that Gustafsson et al. (2008) demonstated that the effects of an $\alpha$-enhancement on the temperature structure in the atmosphere 

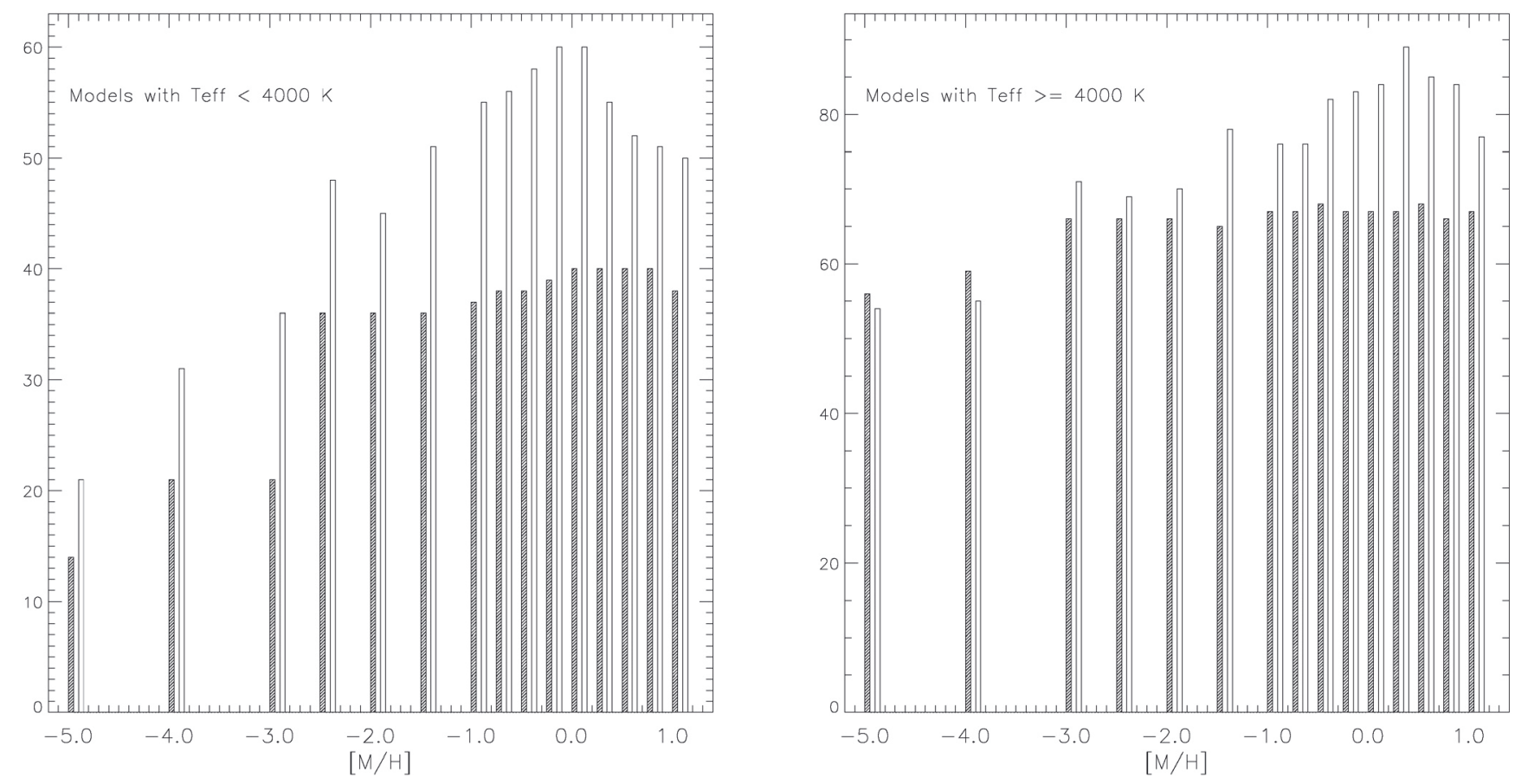

Fig. 1. Distribution of the selected MARCS model atmospheres with respect to the metallicity for effective temperatures cooler (left panel) or hotter (right panel) than $4000 \mathrm{~K}$. Filled and blank histograms refer to the adopted geometry for the radiative transfer, i.e. plane-parallel or spherical, respectively.

become significant below $T_{\text {eff }}<\sim 4500 \mathrm{~K}$ for dwarfs and giants (leading to larger temperature gradients in the deep layers and to higher surface heating, both being mainly caused by $\mathrm{TiO}$ lines). For the present AMBRE grid, at a given metallicity, we only selected the MARCS models with an $[\alpha / \mathrm{Fe}]$ enrichment consistent with the chemical properties of most of the Galactic stars, i.e. $[\alpha / \mathrm{Fe}]=0.0$ for $[\mathrm{M} / \mathrm{H}] \geq 0.0,[\alpha / \mathrm{Fe}]=+0.4$ for $[\mathrm{M} / \mathrm{H}] \leq-1.0$, and $[\alpha / \mathrm{Fe}]=-0.4 \times[\mathrm{M} / \mathrm{H}]$ for $-1.0 \leq[\mathrm{M} / \mathrm{H}] \leq 0.0$.

Regarding the assumed geometry in these calculations (model atmospheres and synthetic spectra), we considered plane-parallel models for $+3.5 \leq \log (g) \leq+5.5$ (in these cases, the atmospheric extension is negligible relative to the radius, thus sphericity effects can be neglected). These models have a microturbulent-velocity parameter set to $1.0 \mathrm{~km} \mathrm{~s}^{-1}$. A spherical geometry was considered when $\log (g) \leq 3.0$ since sphericity effects may be important for low masses and/or low $\log (g)$ (see Heiter \& Eriksson 2006; Gustafsson et al. 2008). For these gravities, a mass of $1.0 M_{\odot}$ and a microturbulence parameter of $2.0 \mathrm{~km} \mathrm{~s}^{-1}$ were considered. Figure 1 presents the distribution of these selected model atmospheres with respect to their metallicity and the adopted plane-parallel/spherical geometry.

On the other hand, we emphasize that all the selected MARCS models were computed assuming a mixing-length parameter $\alpha=l / H_{\mathrm{p}}=1.5$ (Gustafsson et al. 2008) and solar abundances from Grevesse et al. (2007) that reflect the most recent three-dimensional hydrodynamic, local thermodynamic equilibrium (LTE) departures and the latest atomic and molecular data for the solar modelling.

In total, 3358 MARCS models were selected (2115 models with $T_{\text {eff }} \geq 4000 \mathrm{~K}$ and 1243 models with $\left.T_{\text {eff }}<4000 \mathrm{~K}\right)$. We point out that a few models are missing (all the combinations of the atmospheric parameters were not available) owing to the approach to the Eddington flux limit and/or poor convergence of the MARCS code (see Gustafsson et al. 2008). The ranges of the atmospheric parameters of the AMBRE grid are summarised in Table 2 and illustrated in Fig. 2 (see also Fig. 1).

\section{The AMBRE grid of synthetic spectra}

From the atmosphere models presented in the previous section, we computed the final grid that consists of 16783 flux normalised and absolute-flux spectra (10575 spectra have $T_{\text {eff }} \geq$ $4000 \mathrm{~K}$ and 6208 have $T_{\text {eff }}<4000 \mathrm{~K}$; it has to be pointed out that $7 \mathrm{cool}$ star spectra with available model atmospheres are missing because of numerical problems). For reasons of consistency with the MARCS model atmospheres of Gustafsson et al. (2008), the spectra were computed assuming the solar chemical composition of Grevesse et al. (2007) and similar geometry, mass, and microturbulence parameter (see previous section).

In the spectrum calculations, five different $[\alpha / \mathrm{Fe}]$ enrichments were adopted for each selected MARCS model. The adopted $[\alpha / \mathrm{Fe}]$ values vary from -0.4 to +0.4 dex (step of 0.2 dex) with respect to the canonical values corresponding to the original abundances of the selected MARCS models (see Table 2 and Fig. 2, bottom left panel). Therefore, these computations were performed with chemical abundances that are sometimes inconsistent with those adopted in the calculation of the model atmosphere structure. However, for each synthetic spectrum, all continuous and line opacities were recomputed from the model structure with the correct chemical composition (see Sect. 5 for an estimation of the errors caused by this assumption).

The delivered spectra cover the wavelength range between $3000 \AA$ and $12000 \AA$ with a constant wavelength step of $0.01 \AA$ (900000 pixels in total). With the Nyquist-Shannon sampling condition, the adopted spectral sampling corresponds to an equivalent resolving power $(R=\lambda / \Delta \lambda)$ higher than 150000 in the ultraviolet/blue spectral domain to $\sim 600000$ in the near-infrared region. We point out that we decided not to perform any convolution (spectral resolution and instrumental profile, stellar rotation, macroturbulence, ...) for these spectra in order to permit an easy and fast adaptation to the properties of any spectrograph and/or astrophysical application. The adopted spectral domain and sampling indeed cover the wavelength 
P. de Laverny et al.: The AMBRE grid of cool star synthetic spectra

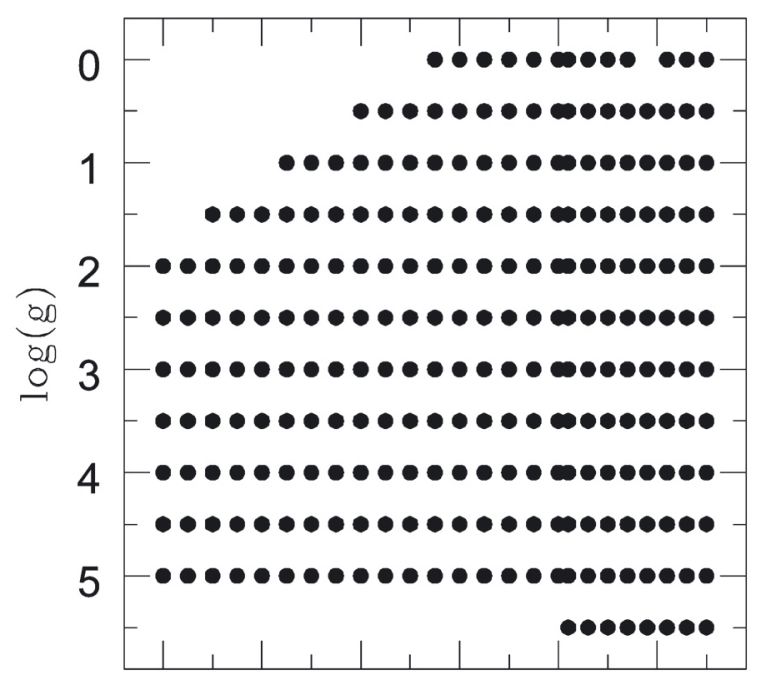

800070006000500040003000

Teff

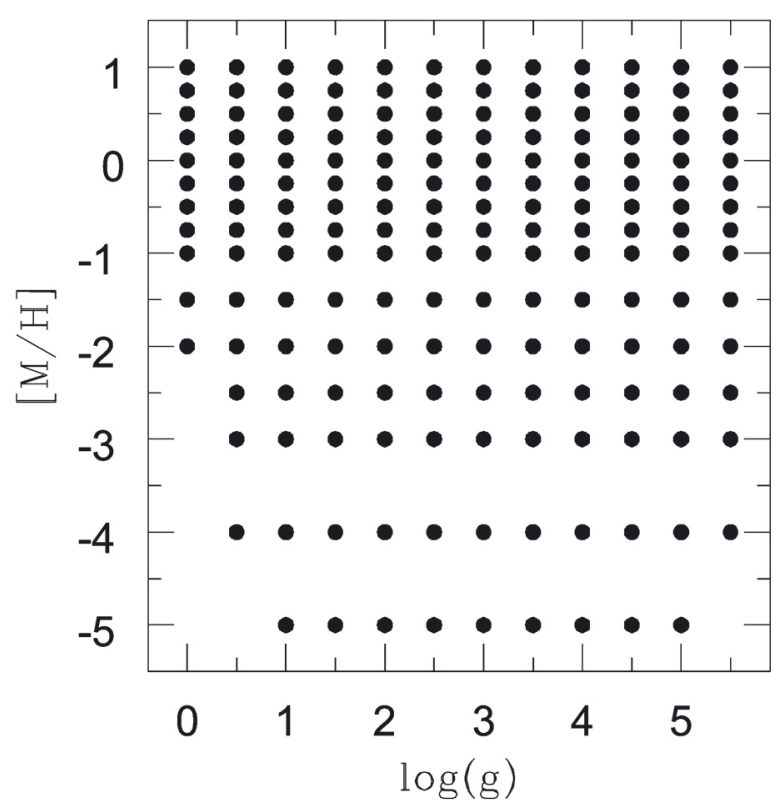

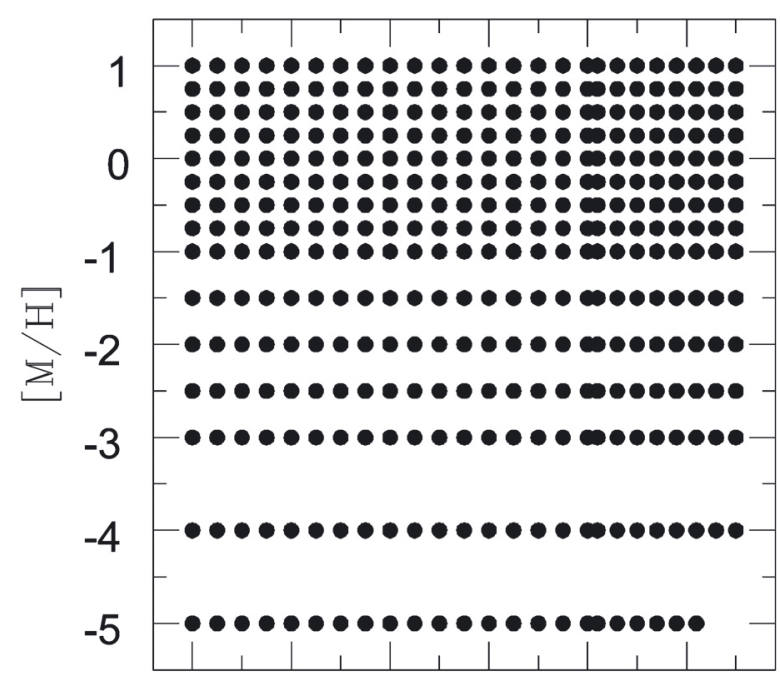

800070006000500040003000

Teff

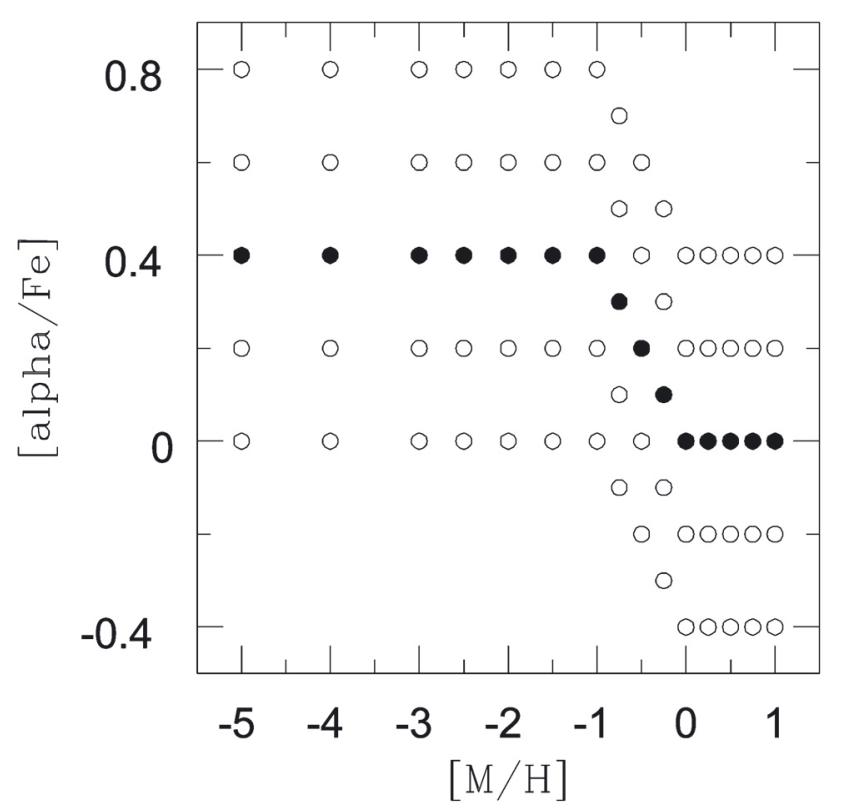

Fig. 2. Distribution of the AMBRE synthetic spectra grid in the atmospheric parameters and $[\alpha / \mathrm{Fe}]$ space. We reiterate that only one value of $[\alpha / \mathrm{Fe}]$ for every $[\mathrm{M} / \mathrm{H}]$ was adopted during the model atmosphere selection process. This is illustrated in the bottom right panel where the AMBRE spectra computed from MARCS models with consistent $[\alpha / \mathrm{Fe}]$ ratios are plotted with filled circles while open circles refer to all the other AMBRE spectra computed with atmosphere models that have inconsistent $[\alpha / \mathrm{Fe}]$ ratios.

ranges explored by all the ESO optical spectrographs, which are the main considerations of the AMBRE project. This spectra grid is also useful for almost any other astronomical optical spectrograph since spectra of any lower resolving power (or largescale broadening) can be easily computed from the provided grid. However, we caution future users of this spectra grid that the adopted wavelength step may be unsuitable for very highspectral resolution applications. The narrowest lines might have indeed been slightly under-sampled in the present grid. This was tested by computing a few spectra with a wavelength step ten times smaller than that of the AMBRE grid (i.e. $0.001 \AA$ ) and then degrading these spectra to different spectral resolutions. Small differences between the spectra with finer sampling and the AMBRE spectra were found for spectral resolutions higher than 50000 (which reached $2-3 \%$ in relative flux in the blue for spectra with $R=100000$ or higher).
All computations were performed in serial mode on the 952 cores (2.4 Ghz Opteron) of the high-performance computing facility SIGAMM of the Observatoire de la Côte d'Azur. The total CPU time used by this project was about $10^{5} \mathrm{~h}$ (which would have been about eleven years if one had used a single computer processing unit!). This long CPU time is almost completely caused by the computation of the opacities produced by the $\sim 10^{7}$ molecular lines in the different atmospheric layers, and it explains why these lines have not been considered in most of the synthetic spectra grids available so far.

Finally, the synthetic spectra are given in terms of absolute surface flux at each wavelength bin in units of erg $/ \mathrm{cm}^{2} / \mathrm{s}^{1} / \AA$ and normalised flux, relative to the local continuum flux (no units). The continuum-normalised spectrum is derived by dividing the absolute flux spectrum by the computed continuum flux in every spectral bin. The size of one spectrum file in FITS format 

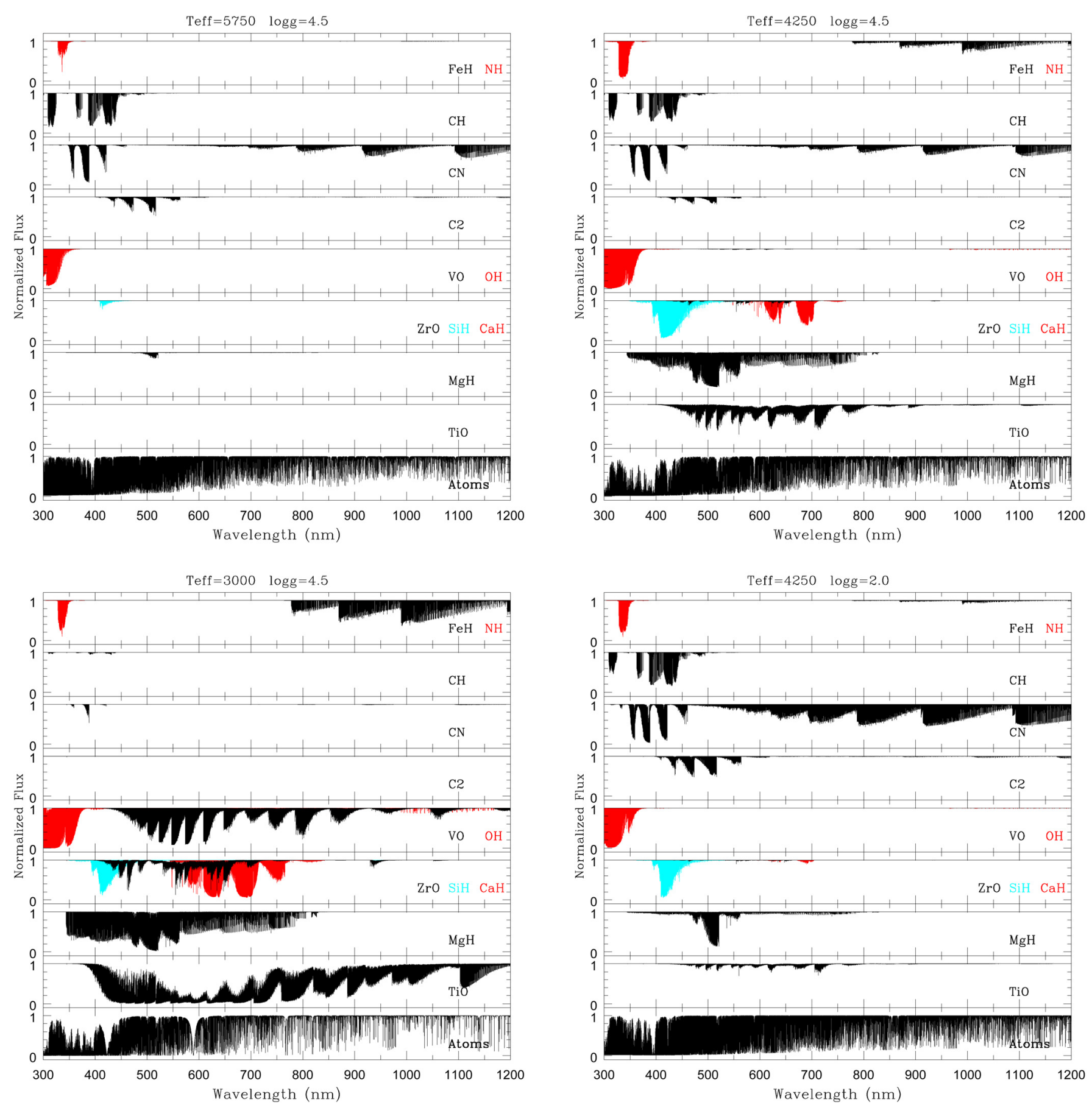

Fig. 3. Normalized spectra computed with only one line specy (all atoms or individual molecules) included in the synthesis. This figure illustrates the line blocking by different species for metal-rich stars with solar $\alpha$-enrichment $([\mathrm{M} / \mathrm{H}]=+0.5$ and $[\alpha / \mathrm{Fe}]=0.0)$. Examples are shown for a solar-type star $\left(T_{\text {eff }}=5750 \mathrm{~K}\right.$ and $\log (g)=4.5$; top left panel $)$, a cool dwarf star $\left(T_{\text {eff }}=4250 \mathrm{~K} \& \log (g)=4.5\right.$; top right panel $)$, a very cool dwarf star $\left(T_{\text {eff }}=3000 \mathrm{~K} \& \log (g)=4.5\right.$; bottom left panel $)$, and a cool giant $\operatorname{star}\left(T_{\text {eff }}=4250 \mathrm{~K}, \log (g)=2.0\right.$; bottom right panel $)$. To ease the comparison, molecules are grouped with respect to their carbon-rich $\left(\mathrm{C}_{2}, \mathrm{CN}\right.$, and $\left.\mathrm{CH}\right)$ or $\alpha$-rich $(\mathrm{TiO}, \mathrm{ZrO}, \mathrm{MgH}, \mathrm{CaH}, \mathrm{SiH}$, and $\mathrm{OH}) \mathrm{nature}$.

is 3.5 Mbytes (about ten times smaller than in ASCII format) and the total grid of 16783 spectra has a size of 59 Gbytes in FITS.

\section{Contribution of the different chemical species to the spectral flux}

To study the contribution of the different chemical species to the total opacity, we computed test spectra by separately considering the different linelists. This analysis was performed on the spectra of different types of stars that are slightly enriched in metals $([\mathrm{M} / \mathrm{H}]=+0.5)$ in order to enhance the different contributions. We show in Fig. 3 the line blocking by different species (atoms and molecules). The atomic lines are the dominant source of line opacity for dwarf and giant stars hotter than $\sim 4000 \mathrm{~K}$. Molecules become the strongest contributors for cooler stars. More precisely, carbon-based diatomic molecules $\left(\mathrm{C}_{2}, \mathrm{CN}\right.$, and $\left.\mathrm{CH}\right)$ make a significant contribution compared to other molecules for $T_{\text {eff }}>4500 \mathrm{~K}$. However, at lower temperatures for dwarf stars, the molecular contribution is dominated 

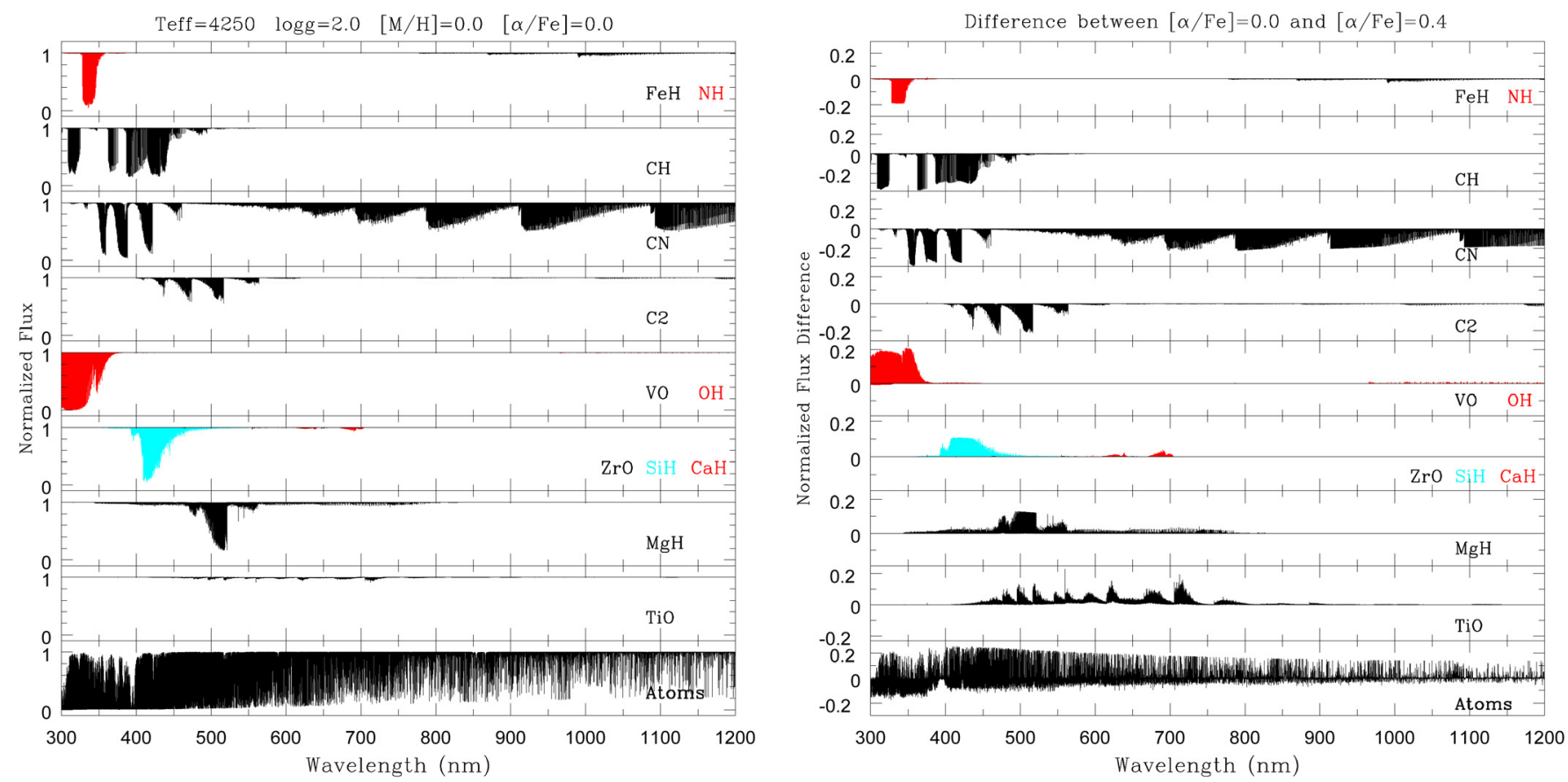

Fig. 4. Spectrum comparison of typical metal-rich giant stars observed towards the Galactic bulge with two different $\alpha$-enhancements. The main atmospheric parameters are $T_{\text {eff }}=4250 \mathrm{~K}, \log (g)=2.0$, and $[\mathrm{M} / \mathrm{H}]=0.0$. Left panel: same as Fig. 3 for normalised spectra computed with only one line specy in the synthesis and assuming $[\alpha / \mathrm{Fe}]=+0.0$ as detected in certain Galactic bulge directions and in the Galactic thin disk. Right panel: line blocking differences between the left panel case $([\alpha / \mathrm{Fe}]=+0.0)$ and a bulge component star having $[\alpha / \mathrm{Fe}]=+0.4$. It can be seen that increasing the abundance of $\alpha$-elements leads to weaker lines of non $\alpha$-atoms and molecules since the continuum flux becomes weaker (see Sect. 4 for more details).
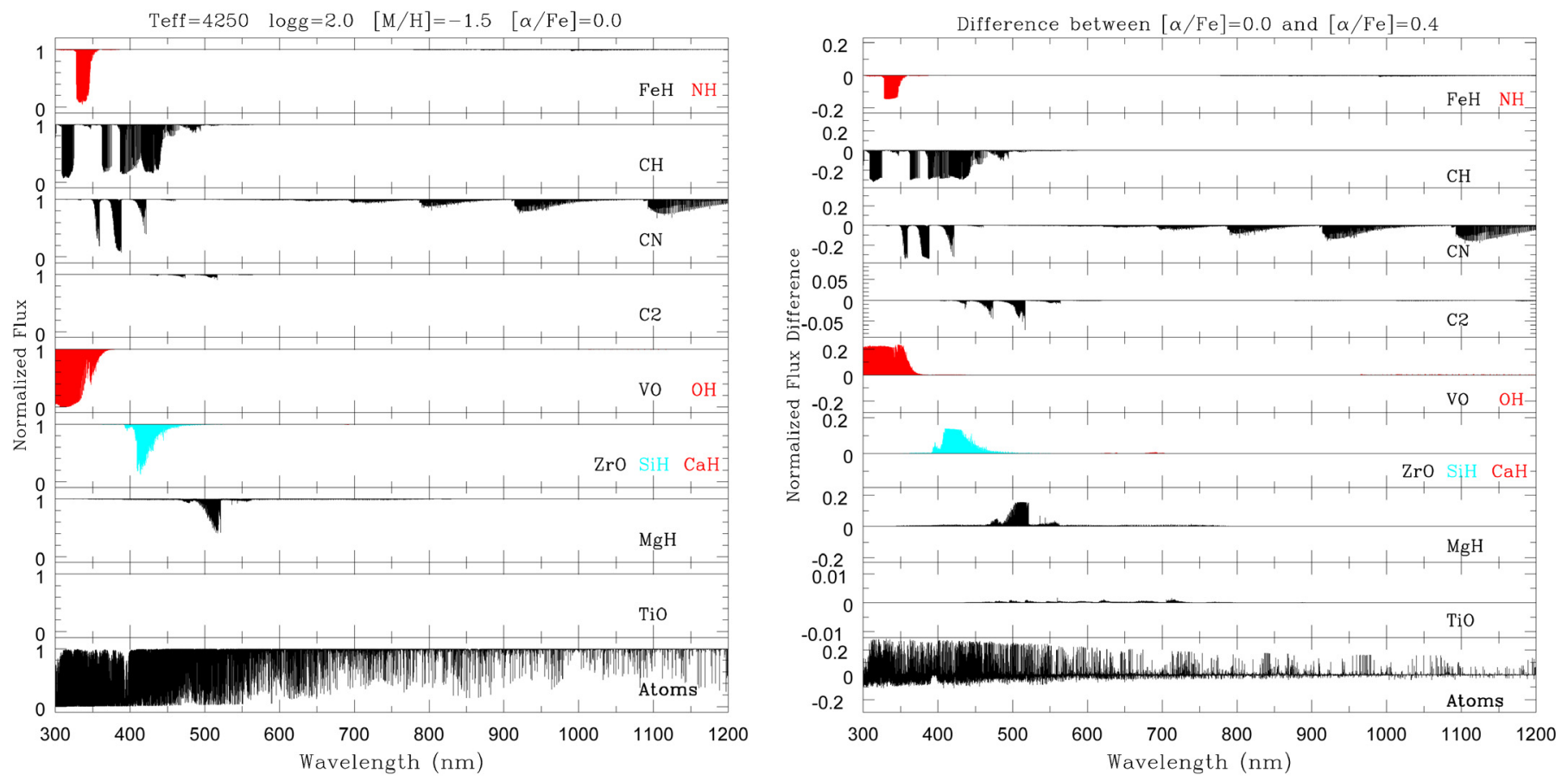

Fig. 5. Same as Fig. 4 for typical metal-poor giant stars of the Galactic halo with two different $\alpha$-enhancements. The main atmospheric parameters are $T_{\text {eff }}=4250 \mathrm{~K}, \log (g)=2.0$, and $[\mathrm{M} / \mathrm{H}]=-1.5$. Left panel: normalised spectra computed with only one line specy in the synthesis and assuming $[\alpha / \mathrm{Fe}]=+0.0$ as detected in halo giants probably originating from accreted dwarf galaxy satellites. Right panel: line blocking differences between the left panel case $([\alpha / \mathrm{Fe}]=+0.0)$ and a giant having $[\alpha / \mathrm{Fe}]=+0.4$ as measured in halo stars formed in-situ (see caption of Fig. 4 , right panel).

by molecules composed of $\alpha$-elements ( $\mathrm{TiO}, \mathrm{ZrO}, \mathrm{VO}, \mathrm{MgH}$, $\mathrm{CaH}, \mathrm{SiH}$, and $\mathrm{OH})$. Their blanketing effect thus strongly increases at these low effective temperatures. These molecules (and TiO in particular) may therefore strongly affect the temperature structure of the atmosphere. In contrast, we note that cool giant spectra are more dominated by carbon-rich molecules than their dwarf counterparts with similar effective temperatures.

We also examined the line blocking of atomic and molecular lines for two typical giant stars of the Galactic bulge and halo (Figs. 4, 5, respectively). In these Galactic components, one 
can encounter stars with slightly different $[\alpha / \mathrm{Fe}]$-enrichments. For that purpose, we therefore computed spectra with similar atmospheric parameters $\left(T_{\text {eff }}, \log (g),[\mathrm{M} / \mathrm{H}]\right)$ and different $\alpha$-enrichments. We point out that in all these comparisons the considered model atmosphere has an $[\alpha / \mathrm{Fe}]$ ratio that is consistent with the one adopted in the synthetic spectrum calculations (this is not the case for every AMBRE spectra, see Sect. 5 for a discussion of this assumption). As we previously mentioned in Sect 2.2, the atmospheric temperature structure of cool dwarfs and giants is strongly affected when the $\alpha$-enhancement is considered (see Gustafsson et al. 2008).

For instance, Fig. 4 compares the spectra of a metal-rich $([\mathrm{M} / \mathrm{H}]=0.0)$ giant of the bulge with $[\alpha / \mathrm{Fe}]=+0.4$ and a typical disk giant $([\alpha / \mathrm{Fe}]=+0.0)$ that could be detected along the same line-of-sight. Similar variations in $\alpha$-enrichments can also be encountered in bulge giants observed at different Galactic latitudes (Gonzalez et al. 2011). It can be seen that differences as large as $10-20 \%$ in relative flux are found for the atomic lines and most molecular lines. These differences affect almost any spectral domain, even those dominated by non- $\alpha$-rich molecules. Furthermore, metallic lines (except those of $\alpha$-elements) and $\mathrm{FeH}, \mathrm{NH}$, or carbon-rich molecular lines appear stronger in the $[\alpha / \mathrm{Fe}]=+0.0$ spectrum. In other words, several lines of non- $\alpha$ elements appear weaker in the $\alpha$-enhanced spectra, although their adopted abundance remains constant. This is because the $\alpha$-elements (and, particularly, $\mathrm{Mg}$, $\mathrm{Si}$, and $\mathrm{Ca}$ ) are important sources of electrons. They therefore strongly contribute to the total continuous opacity, dominated by the $\mathrm{H}^{-}$ion in the studied stars (with respect to HI Rayleigh scattering). Thus, the continuum flux level of $\alpha$-enhanced spectra is lower. For instance, in the two spectra of Fig. 4, the continuum flux is $25 \%$ higher in the blue for the $[\alpha / \mathrm{Fe}]=+0.0$ spectrum than the $[\alpha / \mathrm{Fe}]=+0.4$ one (this difference decreases towards longer wavelengths and is less than $2 \%$ in the nearinfrared). In summary, increasing the abundance of $\alpha$-elements therefore leads to weaker lines of non $\alpha$-atoms and molecules in normalised spectra.

Similar effects (with identical causes) can be seen in Fig. 5 where the differences between two halo giant spectra $([\mathrm{M} / \mathrm{H}]=$ $-1.5)$ are shown: one formed in-situ $([\alpha / \mathrm{Fe}]=+0.4)$ and one possibly accreted from a satellite dwarf galaxy $([\alpha / \mathrm{Fe}]=+0.0$; as found for instance in Fornax or Sculptor, see Tolstoy et al. 2009). It can again be noted that the main differences between both spectra are found for the atomic lines and those of $\mathrm{NH}$, $\mathrm{CH}, \mathrm{CN}$, and $\mathrm{C}_{2}$ (their lines appear deeper in the $[\alpha / \mathrm{Fe}]=+0.0$ spectrum), whereas the $\mathrm{OH}, \mathrm{SiH}$, and $\mathrm{MgH}$ lines appear much stronger for $[\alpha / \mathrm{Fe}]=+0.4$.

As a consequence of all these discussions, we point out again that the integrated spectra of Galactic stellar components or stellar populations found in external galaxies containing large proportions of cool main-sequence and RGB stars is thus affected by their respective enrichments in $\alpha$-elements. This should be carefully considered when interpreting these integrated spectra.

\section{Inconsistent $[\alpha / \mathrm{Fe}]$ ratios between the model atmospheres and the synthetic spectra}

We recall that several spectra of the AMBRE grid were computed from model atmospheres with enrichments in $\alpha$-elements that are inconsistent with those adopted in the spectral synthesis (a difference, denoted as $\Delta[\alpha / \mathrm{Fe}]$ hereafter, of $\pm 0.2 \mathrm{dex}$ or \pm 0.4 dex can be encountered $)^{2}$. This shortcoming was adopted in order to compute a spectra grid with several variations in $[\alpha / \mathrm{Fe}]$ (even if no MARCS models were available) in order to be able, for instance, to automatically derive the $[\alpha / \mathrm{Fe}]$ content of the analysed spectra as required by several galactic archaeology projects. When we recovered the MARCS models in 2009, too few models with several $[\alpha / \mathrm{Fe}]$-values for a given metallicity were available (only some $\alpha$-poor models existed). The situation has since improved because more $\alpha$-enhanced or $\alpha$-poor models have become available since summer 2010 and many more MARCS models should be computed in the near future (as they will be invaluable tools for Galactic surveys such as the Gaia-ESO Survey or Gaia). When these MARCS models become available, our adopted assumption could be easily relaxed when computing a new grid.

Therefore, the AMBRE grid contains spectra for which the continuous and line opacities were computed from $(T, \tau)$-laws with different $[\alpha / \mathrm{Fe}]$ values. Since most of the $\alpha$-elements (such as $\mathrm{Mg}, \mathrm{Si}$, and $\mathrm{Ca}$ ) are important electron-donors, their individual abundances directly affect the number of $\mathrm{H}^{-}$ions (which are a major opacity source in cool stars) formed in the different atmospheric layers and, therefore, the computed atmospheric structure. This assumption will have additional effects since the TiO molecule (composed of two $\alpha$ atoms) strongly affects the atmospheric structure of the coolest models $\left(T_{\text {eff }}<\sim 4500 \mathrm{~K}\right)$ as already mentioned in the previous section. To estimate the consequences of this assumption, we computed the differences between some AMBRE spectra and others computed without adopting this assumption, i.e. with consistent $[\alpha / \mathrm{Fe}]$ ratios in the model atmosphere and the spectrum computation (called $e x$ act spectra, hereafter). We show in Fig. 6 different examples in which the AMBRE spectra were computed with model atmospheres having an $[\alpha / \mathrm{Fe}]$ differing by $\Delta \alpha= \pm 0.2$ dex with respect to the exact spectra. It can be seen that the agreement is very good (differences are smaller than a few percent in normalised flux at any wavelength) for $T_{\text {eff }}>4000 \mathrm{~K}$, regardless the value of the gravity. The largest differences are found below $\lambda<5000 \AA$, where most of the lines are encountered. However, differences as large as $\sim 10 \%$ over the whole optical domain appear for much cooler effective temperatures where many more lines contribute to the spectra. We also checked that these differences, which are probably produced by a continuum level imperfectly estimated in the tested AMBRE spectra, do not strongly vary when the stellar metallicity increases. For metal-poor stars, the agreement is, of course, much better.

The effects of larger differences in $[\alpha / \mathrm{Fe}]$-ratios between the adopted atmospheric structure and the spectral synthesis are shown in Fig. 7 for a rather metal-poor star and a very metalrich one (for which the studied effects are extreme). For rather hot stars $\left(T_{\text {eff }}>4500 \mathrm{~K}\right)$, the spectrum differences are still quite small independently of the luminosity and metallicity. The differences are always smaller than a few percent for $\lambda>5000 \AA$ and stay below $\sim 10 \%$ more in the blue. However, larger differences (up to 10-15\%) are encountered for cooler stars with effective temperatures below $\sim 4000 \mathrm{~K}$. For even cooler stars (M spectral types), the quality of the AMBRE spectra with $\Delta[\alpha / \mathrm{Fe}]= \pm 0.4$ dex between the model atmospheres and the spectral synthesis is rather poor and they should be used very cautiously (or avoided whenever possible).

Furthermore, to quantify the differences caused by the different adopted $[\alpha / \mathrm{Fe}]$ ratios between the model atmospheres

\footnotetext{
2 The tests performed in the previous section do not suffer from this inconsistency.
} 
P. de Laverny et al.: The AMBRE grid of cool star synthetic spectra
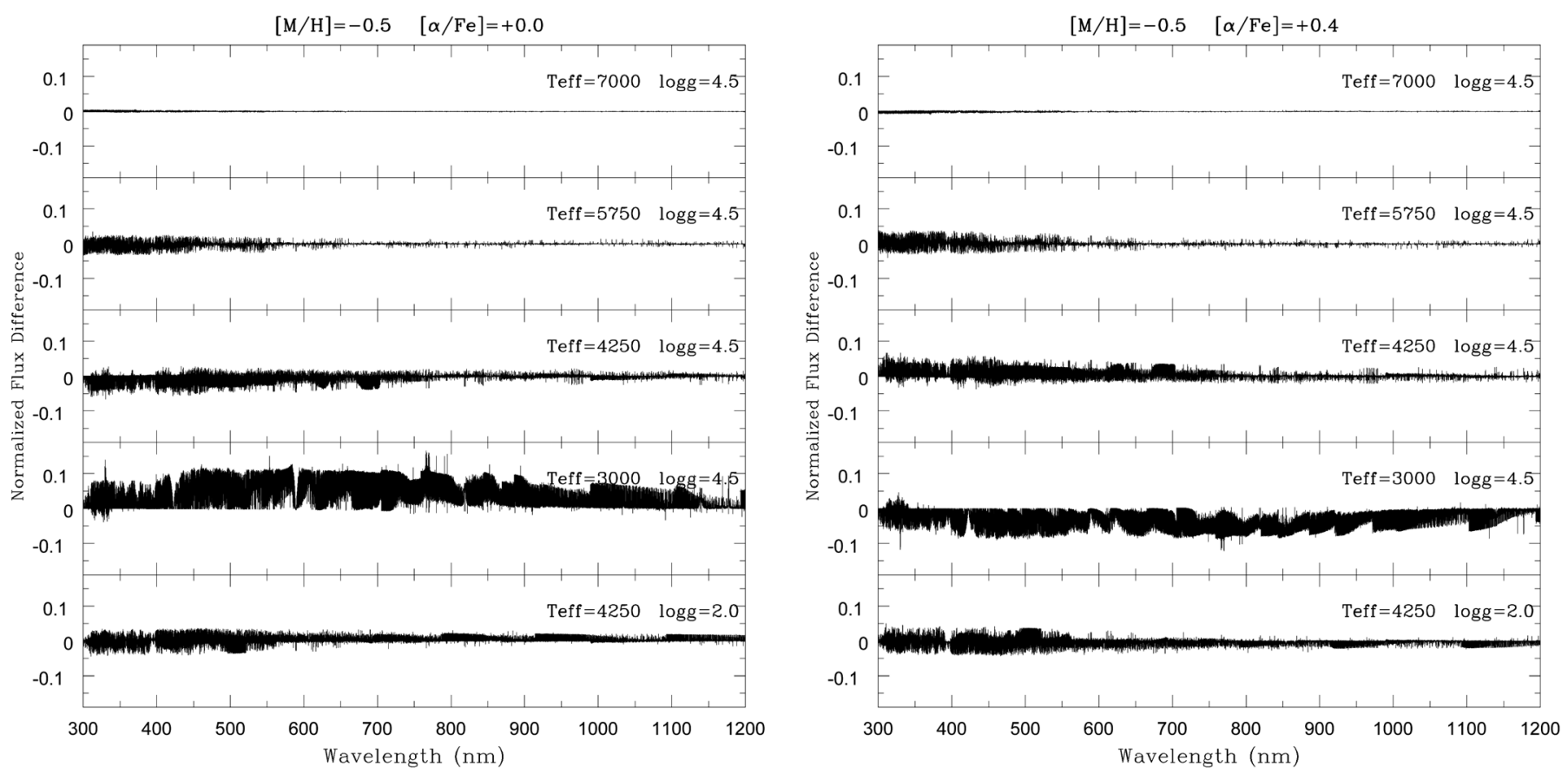

Fig. 6. Differences between the exact spectra (computed by adopting consistent $[\alpha / \mathrm{Fe}]$ ratios in the model atmosphere and the spectral synthesis) and the corresponding AMBRE ones. The computed differences correspond to the exact spectrum minus the corresponding AMBRE spectrum. The $[\alpha / \mathrm{Fe}]$ ratio of the adopted model atmosphere for the AMBRE spectra of the left panel is always +0.2 dex, while the spectral synthesis was performed assuming $[\alpha / \mathrm{Fe}]=+0.0$. In the right panel, the adopted model atmosphere for the AMBRE spectra are for $[\alpha / \mathrm{Fe}]=+0.2$, while we assumed $[\alpha / \mathrm{Fe}]=+0.4$ for the spectral synthesis. The effective temperature and surface gravity of the exact and AMBRE spectra is indicated in the different panels. All spectra (exact and AMBRE ones) have $[\mathrm{M} / \mathrm{H}]=-0.5$ and $[\alpha / \mathrm{Fe}]=+0.0$ or +0.4 as indicated in the figure title (left and right panels, respectively).
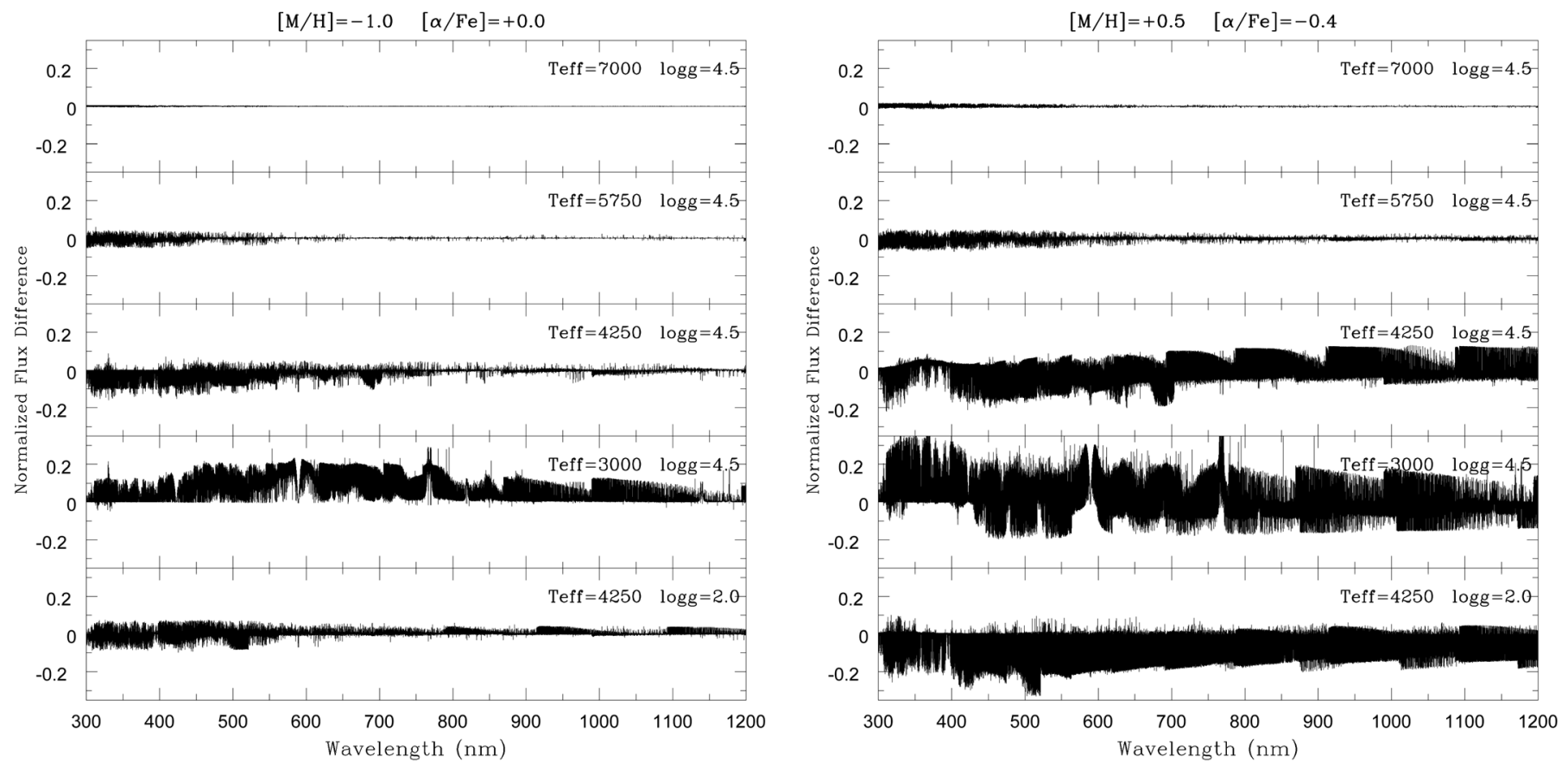

Fig. 7. Same as Fig. 6 for $[\alpha / \mathrm{Fe}]$ of the AMBRE spectra differing by \pm 0.4 dex between the model atmosphere and the spectral synthesis. Left panel: typical metal-poor stars with low $\alpha$-enrichment. The $[\alpha / \mathrm{Fe}]$ ratio of the adopted model atmosphere for the AMBRE spectra is always +0.4 dex, while the spectral synthesis was performed assuming $[\alpha / \mathrm{Fe}]=+0.0$. Right panel: very metal-rich stars with extremely low $\alpha$-enrichment. The adopted model atmosphere for the AMBRE spectra correspond to $[\alpha / \mathrm{Fe}]=+0.0$, while we assumed $[\alpha / \mathrm{Fe}]=-0.4$ for the spectral synthesis presented here.

and the spectral synthesis, we computed the difference using a $\chi^{2}$ estimation between an exact spectrum and several AMBRE ones with close stellar parameters. Practically, we considered AMBRE spectra with $T_{\text {eff }}, \log (g),[\mathrm{M} / \mathrm{H}]$, and $[\alpha / \mathrm{Fe}]$ values within $\pm 1000 \mathrm{~K}, \pm 0.5 \mathrm{dex}, \pm 0.5 \mathrm{dex}$, and $\pm 0.4 \mathrm{dex}$, respectively, around the parameters of the exact spectrum. We thus computed differences between several hundred AMBRE spectra and a given exact one. All the stellar parameter combinations 
of Figs. 6 and 7 were considered. This test enables us to verify whether an algorithm could automatically derive the correct atmospheric parameters of an input spectrum (an exact one in this case) using the AMBRE grid. We found that the correct solution is always recovered if the effective temperature is higher than $\sim 4500 \mathrm{~K}$, whatever the $[\alpha / \mathrm{Fe}]$ enrichment. For cooler stars, when $\Delta[\alpha / \mathrm{Fe}]= \pm 0.2 \mathrm{dex}$ and $T_{\text {eff }}<4000 \mathrm{~K}$, the right solution is also perfectly recovered for dwarf stars, while giants are found to be $\sim 250 \mathrm{~K}$ hotter (one grid step in $T_{\text {eff }}$, all other parameters being accurately retrieved) when $\Delta[\alpha / \mathrm{Fe}]=-0.2$ dex or for a surface gravity slightly higher (one grid step in $\log (g)$, all other parameters being accurately retrieved) when $\Delta[\alpha / \mathrm{Fe}]=$ +0.2 dex. For the coolest cases $\left(T_{\text {eff }}<\sim 3700 \mathrm{~K}\right.$ for dwarfs and giants with $\Delta[\alpha / \mathrm{Fe}]= \pm 0.2$ dex or when $\Delta[\alpha / \mathrm{Fe}]= \pm 0.4 \mathrm{dex}$ and $T_{\text {eff }}<4250 \mathrm{~K}$ ), the closest spectra are always found to be within one step grid in each atmospheric parameters. However, depending on the test cases, any of the four atmospheric parameters could be erroneously derived, although only $T_{\text {eff }}$ or $\log (g)$ are affected in most cases. In the worst cases (very cool stars and $\Delta[\alpha / \mathrm{Fe}]= \pm 0.4 \mathrm{dex})$, two of these parameters can be simultaneously poorly recovered, although always within one step grid. We therefore conclude that, even in these extreme cases (very cool stars and/or large $\Delta[\alpha / \mathrm{Fe}])$ where the accuracy of the parameter determination has decreased, the main stellar properties are still recovered with sufficient accuracy to classify the observed stars. The situation was also found improved when only the reddest part of the spectra $(\lambda>\sim 5500 \AA)$ was considered since a large amount of the atomic and molecular lines are found at shorter wavelengths.

Finally, we point out that similar limitations affect absolute flux spectra. However, most of these differences would be partly or completely hidden for observed spectra with moderate or low signal-to-noise ratios ( $\sim 50$ or lower) and/or very metal-poor stars.

\section{Data access}

One of our goals while computing this grid was to offer it to the whole astronomical community. It can therefore be easily recovered upon request from the authors ${ }^{3}$. Each spectrum (relative and absolute fluxes) can be obtained individually in FITS format (3.6 Mb each). The whole grid (16783 spectra) has a total size of $\sim 60 \mathrm{~Gb}$.

In addition, a large part of this grid has been made publicly available through the POLLUX database of synthetic stellar spectra (Palacios et al. 2010). Every AMBRE spectrum with $5000 \leq T_{\text {eff }} \leq 8000 \mathrm{~K}$ can presently be recovered from the POLLUX website ${ }^{4}$ that contains 7940 AMBRE spectra, i.e. about $50 \%$ of the whole grid.

\section{Conclusion}

We have computed, and made publicly available, a very large grid of synthetic FGKM stellar spectra covering the whole optical domain. It includes almost any possible combination of the atmospheric parameters $T_{\text {eff }}, \log (g),[\mathrm{M} / \mathrm{H}]$, and $[\alpha / \mathrm{Fe}]$ (including extreme metal-poor and metal-rich stars). These spectra are based on (and are mostly consistent with) the MARCS model atmospheres and include the most complete atomic and molecular linelists available so far. We have shown that about $90 \%$

\footnotetext{
3 laverny@oca.eu

${ }^{4}$ http://pollux.graal .univ-montp2.fr/
}

of the provided spectra, including those spectra with extreme $[\alpha / \mathrm{Fe}]$ values that have been computed with $[\alpha / \mathrm{Fe}]$ abundances inconsistent with those of the model atmospheres, can be safely adopted for any use under these criteria: (i) the considered $[\alpha / \mathrm{Fe}]$ ratios differ at maximum by \pm 0.2 dex between the model atmosphere and the spectral synthesis; (ii) high $T_{\text {eff }}(\geq \sim 4250 \mathrm{~K})$ and larger $\Delta[\alpha / \mathrm{Fe}]$ values; or (iii) the most metal-poor stars for any value of $\Delta[\alpha / \mathrm{Fe}]$.

However, when $\Delta[\alpha / \mathrm{Fe}]= \pm 0.4$ dex and $T_{\text {eff }}<\sim 4250 \mathrm{~K}$, the spectra should be considered very cautiously (or avoided when possible), although they are still similar enough to the exact ones (within one step grid in every parameter dimension) to allow spectral classification.

We are well aware that these "classical" model atmospheres and synthetic spectra can be questioned when they are carefully compared to real stars, since the effects of the adopted different assumptions (LTE, 1D, static atmospheric structure, varied abundances of individual elements, ...) could be large for some specific cases. The accuracy of the adopted linelist data together with their systematics should also be questioned. For instance, for the coolest spectra of this grid (below $\sim 2800 \mathrm{~K}$ ), we considered neither the model atmospheres nor the opacities caused by the formation of dust grains. This assumption may at least affect the red part of the spectra and/or metal-rich models (see Chabrier et al. 2000). However, several of these effects could be partly corrected by adopting specific calibrations defined with rather well-known reference stars (see, for instance, the procedure adopted within the AMBRE project; Worley et al. 2012). Furthermore, more advanced models (including NLTE effects, $3 \mathrm{D}$, hydrodynamics, ...) are pratically incapable of covering the whole range of stellar parameters considered in this work, and there exists no other alternative than to compute such "classical" grids.

However, the grid that we provide consists of high resolution spectra. It can be very easily adapted to any real observed spectra, either by slicing them over the covered wavelength ranges, convolution with a well-adapted kernel, and/or adopting a specific sampling law (see the application to the analysis of FEROS spectra in Worley et al. 2012). We also preferred not to consider any stellar rotational velocities and/or macroturbulence profiles in order to ensure a very broad use. This grid is the basis of the whole AMBRE project, which consists of the analysis of most of the ESO high-resolution archive spectra. All of the training phases of the parametrisation algorithms have indeed been performed with these AMBRE spectra, phases that have been adapted in turn to each of the four selected spectrographs including their different instrument set-ups. This grid has also been adopted for several tests of the classification algorithms developed for the Gaia/RVS spectra and for the preparation of the Gaia-ESO Survey (selection of the GIRAFFE setups, estimates of the uncertainties in the derived atmospheric parameters and abundances, ...). Finally, other applications could easily be anticipated such as, for instance, the study of the integrated spectra of the different Galactic and extragalactic components, including those with multiple populations, and the dependences of their different physical properties with respect to the stellar $[\mathrm{M} / \mathrm{H}]$ and/or $[\alpha / \mathrm{Fe}]$ distributions.

Finally, the next step in compiling similarly large spectra grids would be to overcome the assumption adopted in the present study that consisted in computing synthetic spectra with extreme $[\alpha / \mathrm{Fe}]$ values that are inconsistent with the available model atmospheres. The consideration of even more complete molecular linelists (including polyatomic molecules) would also be of great interest for the coolest spectra of the present grid. 
We also point out that an extension to hotter $T_{\text {eff }}$ with ATLAS12 Kurucz stellar atmosphere models is planned for the future, adopting the same linelists (extended towards higher ionisation stages) and consistent solar abundances in order to maintain a high consistency between different spectra grids covering a much larger range of effective temperatures.

Acknowledgements. The AMBRE project has been supported by the Centre National d'Études Spatiales (CNES), the European Southern Observatory, and the Observatoire de la Côte d'Azur (OCA). The spectra calculations were performed with the high-performance computing facility SIGAMM, hosted by OCA. We acknowledge the Vienna Atomic Line Database (VALD) for its compilation of atomic line parameters. We sincerely thank B. Edvardsson for his encouraging remarks on this paper and the stellar atmosphere group in Uppsala for providing the MARCS model atmospheres to the community.

\section{References}

Alvarez, R., \& Plez, B. 1998, A\&A, 330, 1109

Barklem, P., Piskunov, N., \& OMara, B. J. 2000a, A\&A, 363, 1091

Barklem, P., Piskunov, N., \& OMara, B. J. 2000b, A\&AS, 142, 467
Chabrier, G., Baraffe, I., Allard, F., \& Hauschildt, P. 2000, ApJ, 542, 464 Coelho, P., Barbuy, B., Meléndez, J., et al., 2005, A\&A, 443, 735

Frebel, A., Kirby, E. N., \& Simon, J. D. 2010, Nature, 464, 72

Gonzalez, O. A., Rajkuba, M., Zoccali, M., et al. 2011, A\&A, 530, A54

Grevesse, N., Asplund, M., \& Sauval, A. J. 2007, Space Sci. Rev., 130, 105

Gustafsson, B., Bell, R. A., Eriksson, K., \& Nordlund, A. 1975, A\&A, 42, 407

Gustafsson, B., Edvardsson, B., Eriksson, K., et al. 2008, A\&A, 486, 951

Heiter, U., \& Eriksson, K. 2006, A\&A, 452, 1039

Kirby, E. N. 2011, PASP, 123, 531

Kordopatis, G., Recio-Blanco, A., de Laverny, P., et al. 2011, A\&A, 535, A106

Kupka, F., Piskunov, N. E., Ryabchikova, T. A., et al. 1999, A\&AS, 138, 119

Letarte, B., Hill, V., Tolstoy, E., et al. 2010, MNRAS, 400, 1472

Munari, U., Sordo, R., Castelli, F., \& Zwitter, T. 2005, A\&A, 442, 1127

Palacios, A., Gebran, M., Josselin, E., et al. 2010, A\&A, 516, A13

Plez, B. 2011, J. Phys. Conf. Ser., 328, 2005

Prugniel, P., \& Soubiran, C. 2001, A\&A, 369, 1048

Recio-Blanco, A., Bijaoui, A., \& de Laverny, P. 2006, MNRAS, 370, 141

Soubiran, C., Le Campion, J.-F., Cayrel de Strobel, G., et al. 2010, A\&A, 515, A111

Tolstoy, E., Hill, V., \& Tosi, M. 2009, ARA\&A, 47, 371

Vazdekis, A., Sánchez-Blázquez, P., Falcón-Barroso, J., et al. 2010, MNRAS, 404,1639

Worley, C. C., de Laverny, P., Recio-Blanco, A., et al. 2012, A\&A, 542, A48

Wu, Y., Singh, H. P., Prugniel, P., et al. 2011, A\&A, 525, A71 